\title{
PELARGONIUM SIDOIDES DC. (UMCKALOABO): HISTÓRICO DO USO, ASPECTOS TAXONÔMICOS, ECOLÓGICOS, FITOQUÍMICOS, FARMACOLÓGICOS E TOXICOLÓGICOS
}

\author{
PELARGONIUM SIDOIDES DC. (UMCKALOABO): USAGE HISTORY, \\ TAXONOMIC, ECOLOGICAL, PHYTOCHEMICAL, PHARMACOLOGICAL AND \\ TOXICOLOGICAL ASPECTS
}

\author{
Bruna Mazzuco ${ }^{1}$ \\ Samara Fenilli Bristot ${ }^{2}$ \\ Silvia DalBó s.4,5 $^{3,4}$ \\ Patrícia de Aguiar Amaral $3,4,5$ \\ Vanilde Citadini-Zanette ${ }^{2,4,5}$ \\ Angela Erna Rossato 3,4
}

\begin{abstract}
RESUMO
Considerando que Pelargonium sidoides DC. é usado tradicionalmente na África do Sul e na Alemanha para infecção das vias respiratórias, incluindo sinusite e tuberculose, além de apresentar baixo potencial de efeitos adversos, tanto para adultos como para crianças, objetivou-se realizar um levantamento bibliográfico observando as publicações disponíveis nas bases Google Acadêmico, Biblioteca Virtual em Saúde (BVS), Portal CAPES, PUBMED, MEDLINE, publicadas nos últimos 20 anos sobre os aspectos etnobotânicos, taxonômicos, ecológicos, fitoquímicos, farmacológicos e toxicológicos da espécie, na perspectiva de fornecer informações para sua utilização segura e racional. Constatou-se que o extrato da planta já é validado como medicamento pela ANVISA, sua validação contribui positivamente para as indicações da espécie na prática médica e representa uma alternativa valiosa devido ao seu efeito antibacteriano e por reforçar a imunidade não específica, consequentemente pode contribuir positivamente para a redução das prescrições de antibióticos desnecessários nas infecções virais das vias aéreas superiores e diminuir os riscos de resistência bacteriana na prática clínica.
\end{abstract}

Palavras-chave: Pelargonium sidoides DC. Planta Medicinal. Fitoterapia.

\begin{abstract}
\footnotetext{
${ }^{1}$ Egressa do Curso de Farmácia, UNESC

${ }^{2}$ Herbário Pe. Dr. Raulino Reitz (CRI), E-mail: aerossato@gmail.com UNESC

${ }^{3}$ Grupo de Extensão e Pesquisa em Assistência Farmacêutica (GEPAF), UNESC

${ }^{4}$ Grupo de Estudos de Pesquisa em Plantas Medicinais (GEPPLAM), UNESC

${ }^{5}$ Programa de Pós-Graduação em Ciências Ambientais (PPGCA), UNESC
}

Whereas Pelargonium sidoides DC. is traditionally used in South Africa and used in Germany for respiratory tract infections, including sinusitis and tuberculosis and has a low potential of adverse effects, for both adults and for children, this paper aimed to perform a bibliographic review about the publications available in Google Scholar, Virtual Health Library (VHL), CAPES Portal, PUBMED, MEDLINE databases, published in the last 20 years on the ethnobotanical, taxonomic, ecological, 
phytochemical, pharmacological and toxicological aspects of the species with a view to providing information for the its safe and rational use. It was found that the extract of the plant has been validated as a medicine by ANVISA. It validation contributes positively for the species indications in medical practice and is a valuable alternative due to its antibacterial effect and enhance non-specific immunity. Consequently it can contribute positively for the decrease unnecessary antibiotics prescription for viral infections of the upper airways and to for decreasing the risks of bacterial resistance in clinical practice.

Keywords: Pelargonium sidoides DC. Medicinal plant. Phytotherapy.

\section{INTRODUÇÃO}

Há milhares de anos a utilização de plantas com fins medicinais vem sendo utilizadas pela humanidade para tratamento, cura e prevenção de doenças. Além da comprovação da ação terapêutica de várias plantas popularmente utilizadas, a fitoterapia representa componente importante da cultura de um povo, sendo também parte de um saber utilizado e difundido pelas populações ao longo de várias gerações (TOMAZZONI et al., 2006).

O emprego correto de plantas para fins terapêuticos pela população em geral, requer o uso de plantas medicinais selecionadas por sua eficácia e segurança, baseadas na tradição popular ou cientificamente validadas como medicinais (LORENZI; MATOS, 2002). Segundo Simões (2010), as descobertas das substâncias ativas presentes nas plantas medicinais alavancaram junto com o início da síntese orgânica, uma revolução científica e tecnológica, alterando muito rapidamente o arsenal terapêutico.

Atualmente, os fitoterápicos são amplamente utilizados em diversos países e no Brasil sua segurança de uso e suas indicações terapêuticas podem ser validadas por levantamentos etnofarmacológicos de utilização, documentações técnico-científicas em publicações ou ensaios clínicos fase 3 (BRASIL, 2014).

O mercado mundial fitoterápico é de US\$ 43 bilhões por ano e somente nos Estados Unidos da América representa US\$ 5 bilhões por ano, sendo o setor que mais cresce no mercado farmacêutico norte-americano. Na África, por exemplo, 80\% da população dependem das plantas medicinais e dos fitoterápicos e estes representam uma alternativa terapêutica frente ao alto custo dos fármacos sintéticos (TUROLLA; NASCIMENTO, 2006; ASCHWANDEN, 2001). 
Dentre as plantas utilizadas na África para fins medicinais e já utilizadas como medicamento fitoterápico, destaca-se Pelargonium sidoides DC., popularmente conhecida como Umckaloabo, indicado no tratamento de infecções agudas ou crônicas do trato respiratório, ouvido e garganta (KIRK; NETTO, 2007) e recentemente introduzido no mercado farmacêutico brasileiro (BRASIL, 2007; BRASIL, 2004).

Diante do exposto, este artigo teve como objetivo realizar levantamento bibliográfico sobre $P$. sidoides, abordando histórico do uso, aspectos taxonômicos, ecológicos, fitoquímicos, farmacológicos e toxicológicos.

\section{METODOLOGIA}

Foi realizado levantamento bibliográfico observando as publicações disponíveis nas bases Google Acadêmico, Biblioteca Virtual em Saúde (BVS), Portal CAPES, Pubmed e MEDLINE, publicadas nos últimos 20 anos sobre Pelargonium sidoides DC. Para a localização foi utilizado o nome científico da planta acrescido dos aspectos propostos e usados os descritores referentes à Pelargonium sidoides. Os critérios para seleção e inclusão, definidos a priori, foram somente artigos e textos disponibilizados na íntegra em português, inglês ou espanhol que abordavam os aspectos etnobotânicos, taxonômicos, ecológicos, fitoquímicos, farmacológicos e toxicológicos. A seleção dos artigos foi com base na relevância das informações apresentadas.

\section{Histórico do uso e características botânicas}

No ano de 1897, o inglês Charles Henry Stevens após adoecer de tuberculose, aconselhado por seu médico, foi à África a fim de curar a doença em climas mais quentes e lá, por intermédio de um curandeiro nativo, conheceu a planta Pelargonium sidoides, preparada e administrada na forma de um "cozido de raiz", conhecido localmente como "Umckaloabo". Após três meses de tratamento, Stevens considerandose curado e convicto do sucesso da cura, introduziu a planta na Inglaterra como alternativa de tratamento para tuberculose (BLADT; WAGNER, 2007). 

impressa) ISSN 2358-9426 (versão eletrônica)

O nome popular "umckaloabo", deriva da língua Zulu da África do Sul, onde umkhulkane significa queixas relacionadas com doenças pulmonares e uhlabo quer dizer dor no peito (KOLODZIEJ, 2008).

P. sidoides pertence à família Geraniaceae, ordem Geraniales e ao clado Rosideas II (APG III, 2009) e é nativa da África do Sul. O gênero Pelargonium L'Hér. ex Aiton compreende mais de 270 espécies de herbáceas a arbustos pequenos, limitados em sua distribuição geográfica, das quais cerca de $80 \%$ ocorrem no sul da África (KOLODZIEJ, 2007). Também ocorrem na Austrália, Nova Zelândia e no Extremo Oriente (MATIVANDLELA et al., 2006).

As espécies do gênero Pelargonium crescem geralmente em campos baixos e algumas vezes entremeados com arbustos e árvores, em solos rochosos que variam de arenoso a argiloso e também em solos xistoso ou basáltico. As plantas são perenes quando cultivadas, mas morrem na natureza durante os períodos secos e no inverno (MATIVANDLELA et al., 2006).

P. sidoides possui consistência herbácea, com folhas longo-pecioladas, concentradas na base da planta; apresenta raízes subterrâneas e espessas; o caule na floração é aveludado com cerca de 20 a $50 \mathrm{~cm}$ de altura, sustentando folhas cordiformes em toda sua extensão. Em sua região de origem é encontrada desde o nível do mar até altitudes de $2300 \mathrm{~m}$ (WHITE, 2007).

$P$. sidoides DC. é facilmente confundida com P. reniforme Curt., pois ambas espécies são muito semelhantes na aparência e forma. No entanto, algumas características as diferem: as sépalas na $P$. sidoides são verdes com margens brancas e as de $P$. reniforme são vermelhas com margens em rosa (WHITE, 2007). As flores de $P$. sidoides se caracterizam pela cor vermelho-escuro a preto, com folhas cordiformes e pólen de coloração verde-amarelado. Em contrapartida, as flores de P. reniforme são vermelhas com marcação em preto e as folhas tendem mais para reniforme do que para cordiforme e o pólen apresenta coloração branco-esverdeado (BLADT; WAGNER, 2007). As pétalas de $P$. sidoides variam de marrom a preto e as de $P$. reniforme de rosa a roxo, conforme demonstrados na figura 1 (WHITE, 2007). 


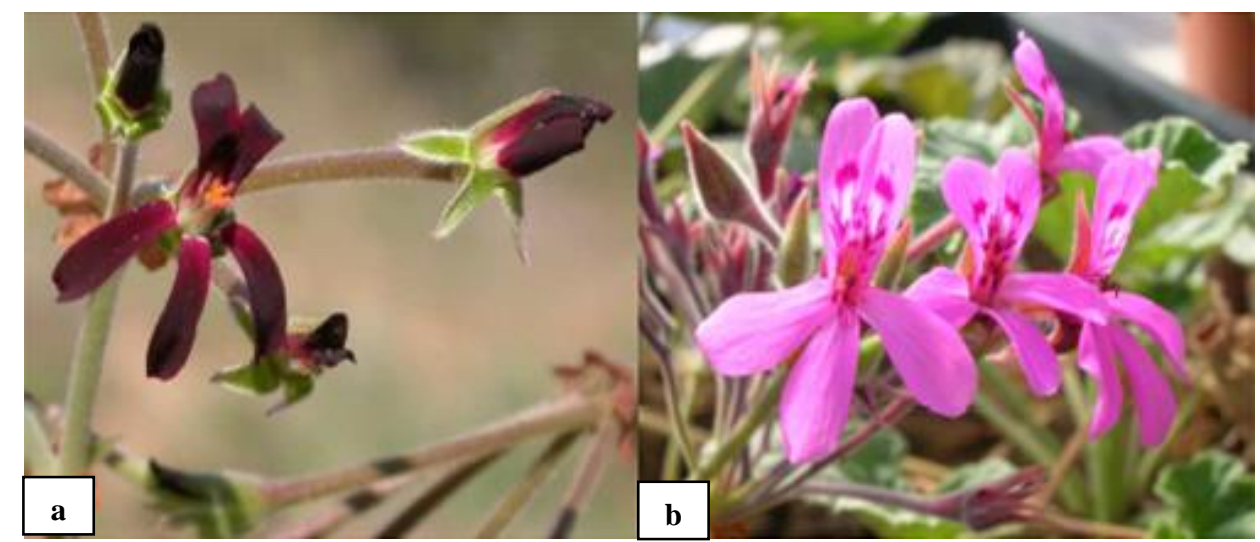

Figura 1: Flores de Pelargonium sidoides DC. (a) e de P. reniforme Curt. (b) Fonte: WHITE (2007).

\section{Características ecológicas}

Pelargonium sidoides, em geral, é uma planta bastante resistente e prefere sol pleno (VAN DER WALT, 1988). A espécie tolera ambientes parcialmente perturbados, mas em situações onde há altos níveis de competição entre espécies invasoras e a presença de atividades agrícolas, a espécie não consegue se desenvolver e regenerar novamente (VLOK, 2003). O cultivo pode ser feito por propagação sexuada e assexuada, isto é, pelo plantio das sementes ou pelo transplante de estacas basais (VAN DER WALT, 1988).

Em seu habitat original, África do sul, P. sidoides enfrenta condições extremas de temperaturas, por isso a espécie possui adaptação àquelas condições: suas partes subterrâneas são bem desenvolvidas o que a possibilita suportar condições adversas para seu desenvolvimento (VAN DER WALT, 1988).

Quanto ao seu status de conservação é classificada na Lista Vermelha de Plantas da África do Sul como Pouco Preocupante (LC), mas sua população está em declínio e sofre ameaça pela degradação do habitat por atividades agropastoris (CASTRO et al., 2012).

\section{Droga vegetal, extrato e principais constituintes químicos.}


O extrato da raiz de $P$. sidoides é muito utilizado pelos praticantes tradicionais e pela população nativa do sul da África em virtude de suas propriedades curativas (KOLODZIEJ et al., 2003).

Comercialmente o extrato de P. sidoides, conhecido como EPs 7630, é produzido pela ISO-Arzneimittel na Alemanha sobre a licença de Dr. Schwabe (KOLODZIEJ et al., 2003) e seu processo de obtenção se dá pela extração das raízes moídas em 11\% (m/m) de etanol em água (SCHOETZ et al., 2008 ).

Os estudos apontam que, o extrato é dominado pela presença de grandes compostos cumarínicos oxigenados, dos quais já foram identificados 10 compostos. Também foram encontrados simples fenóis e ácido gálico, sendo estes considerados os principais metabólitos do vegetal; outros componentes incluem taninos, flavonóides, fitosteróis e ácidos graxos insaturados (KOLODZIEJ et al., 2003; SEIDEL; TAYLOR, 2004). Além disso, uma considerável proporção de proantocianidina de alto peso molecular foi encontrada no EPs 7630 (KOLODZIEJ 2007; SCHOTZ; NOLDNER, 2007).

Taninos e diferentes compostos cumarínicos, entre eles umckalin que é típico desta planta, parecem estar relacionados com as propriedades imunomoduladoras e antibacterianas (GOLOVATIOUK; CHUCHALIN, 2002).

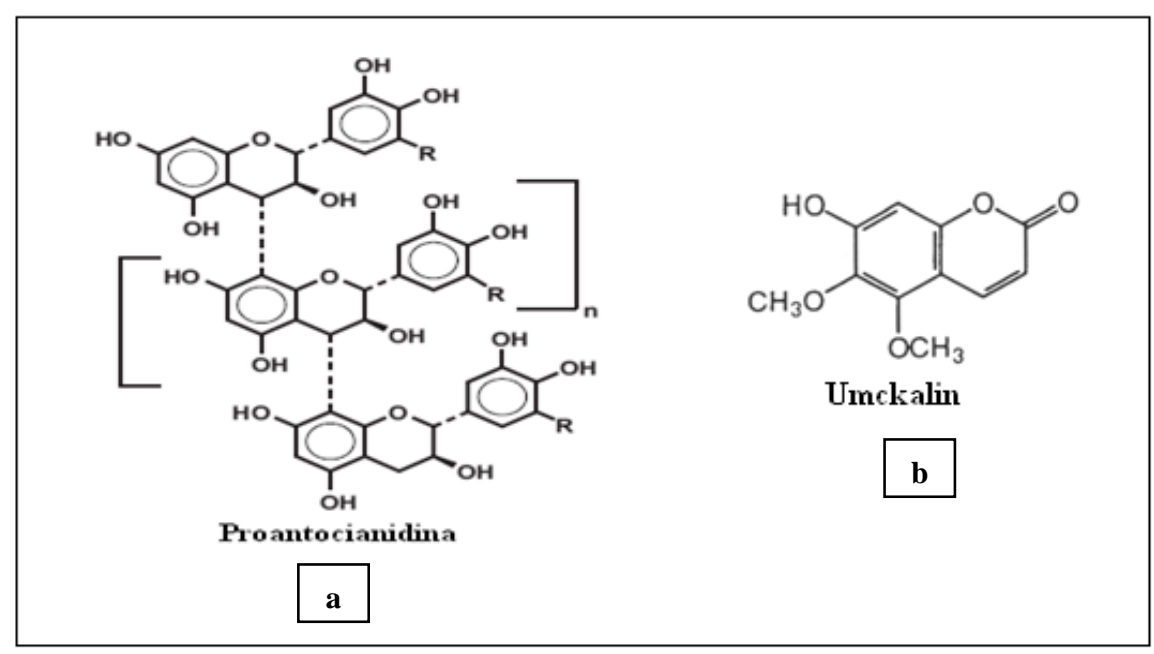

Figura 2: Principais estruturas químicas componentes do Pelargonium sidoides:

Proantocianidina (Tanino condensado) (a) e Umckalin (Cumarína) (b).

Fonte: KOLODZIEJ (2007). 
A ocorrência de taninos pode explicar o tradicional uso das partes aéreas como agente de cicatrização e também pode ser atribuído à sua ação adstringente (KOLODZIEJ, 2007).

Embora estudos ainda estejam em andamento, os derivados cumarínicos e compostos fenólicos, incluindo ácidos fenólicos e proantocianidina são os principais compostos encontrados no extrato EPs 7630 (KOLODZIEJ, 2007; KOCH; BIBER 2007) e este é padronizado para conter $0,08 \%$ a $0,32 \%$ de fenóis totais (marcador químico) (ADIS INTERNATIONAL LTDA, 2006).

\section{Indicações Terapêuticas}

Preparações a base de raízes de $P$. sidoides têm sido tradicionalmente utilizado na medicina popular da África do Sul para o tratamento de diversas enfermidades como diarréia, dismenorréia, distúrbios hepáticos e infecção das vias respiratórias, incluindo sinusite e tuberculose. Também são citados tratamento de fadiga, ouvido, garganta, febre e fraqueza generalizada (LEWU et al., 2006; VAN WYK, 2008; KOLODZIEJ, 2007).

Na medicina popular a utilização das partes aéreas de $P$. sidoides para tratamento de doenças gastrointestinais, tais como diarréia e cicatrização de feridas, estão relacionadas ao teor elevado de proantocianidina. $\mathrm{E}$ os efeitos relacionados com a cura de distúrbios hepáticos estão relacionados com a alta gama de compostos fenólicos que a raiz de $P$. sidoides possui (KOLODZIEJ; KIDERLEN, 2007).

A partir de 1933 o extrato de $P$. sidoides foi introduzido na Alemanha e teve seu uso difundido, sendo considerado um medicamento da moderna fitoterapia alemã (KIRK; NETTO, 2007) e atualmente o extrato de P. sidoides (EPs 7630), vem sendo utilizado principalmente para tratar infecções do trato respiratório (SCHÖTZ; NÖLDNER, 2007) e bronquite aguda (SCHNITZLER et al., 2008).

A partir de estudos farmacológicos realizados na década de 70, houve embasamento científico para as indicações do uso de $P$. sidoides no tratamento das infecções respiratórias, sendo comprovados os seus efeitos antimicrobianos e imunomoduladores, embora ainda não esteja totalmente esclarecido seu mecanismo de 

impressa) ISSN 2358-9426 (versão eletrônica)

ação. Estudos farmacocinéticos ainda não foram realizados, devido à complexa composição do extrato da planta (KIRK; NETTO, 2007).

No Brasil a Agência Nacional de Vigilância Sanitária (ANVISA) aprovou medicamentos fitoterápicos com esta planta, dentre eles Umckan ${ }^{\circledR}$ e Kaloba ${ }^{\circledR}$ ambos elaborados a partir das raízes de P. sidoides (BRASIL, 2007; BRASIL, 2004).

\section{Estudos de Atividade Farmacológica}

\section{Bronquite Aguda}

A Bronquite Aguda é definida como uma infecção do trato respiratório inferior e é um dos diagnósticos mais comuns feitos pelos médicos na atenção primária à saúde (MATTHYS et al., 2003). O interesse em tratá-la com raízes de P. sidoides (EPs 7630) é devido aos efeitos antibacterianos e imunomoduladores desta planta, demonstrados em vários ensaios clínicos. As substâncias naturais mais importantes e responsáveis pela ação farmacológica, neste caso, são os compostos polifenólicos (taninos) e simples compostos fenólicos cumarínicos (AGBABIAKA et al., 2008).

O provável mecanismo de ação é atribuído à capacidade que o EPs 7630 tem em antagonizar a aderência bacteriana e/ou invasão dos epitélios intactos, protegendo assim as vias respiratórias superiores contra a colonização de bactérias nas infecções (AGBABIAKA et al., 2008).

Em um estudo multicêntrico, prospectivo, aberto observacional, foi investigado a eficácia e segurança da solução de EPs 7630 no tratamento de bronquite aguda. O total de pacientes tratados foi de 2099, que cumpriram os critérios de inclusão, apresentando tosse produtiva por menos de seis dias, sem nenhuma indicação para o tratamento com antibióticos. Os pacientes foram instruídos a tomar três vezes ao dia o EPs 7630, 30 minutos antes das refeições, durante um período de 14 dias (MATTHYS et al., 2007).

Os resultados deste estudo demonstraram boa eficácia e segurança do EPs 7630, no tratamento de bronquite aguda em crianças e adultos. Recuperação completa ou grande melhoria dos sintomas foram de $94,2 \%$ de todos os pacientes, a tolerabilidade de EPs 7630 foi classificada como muito boa, os eventos graves não foram relatados e a taxa de eventos adversos foi de 1,2\% (MATTHYS et al., 2007). 

impressa) ISSN 2358-9426 (versão eletrônica)

Já Golovatiouk e Chuchalin (2002), conduziram um estudo randomizado, duplo cego e controlado por placebo em 124 pacientes durante 12 meses em seis policlínicas em Moscou. Os pacientes tinham que ser maior de 18 anos e sofrer de bronquite aguda, sendo a duração média do tratamento de sete dias. A administração do EPs 7630 e a solução contendo Placebo foram de 30 gotas por dia antes das refeições, ambas as substâncias ativas e o placebo eram indistinguíveis na aparência. Dos 124 pacientes incluídos no estudo, 64 deles (15 homens e 49 mulheres) ingeriram EPs 7630 e 60 (22 homens e 38 mulheres) ingeriram o placebo. Os resultados indicaram que EPs 7630 foi significativamente melhor do que o placebo. Eventos adversos foram relatados em 15 dos 64 pacientes tratados com EPs 7630 e em 10 dos 60 doentes tratados com o placebo, e nenhum destes eventos foi classificado como grave. A tolerabilidade de EPs 7630 foi avaliada como muito boa por $98,4 \%$ dos pacientes e $96,7 \%$ dos médicos. A eficácia clínica do extrato de $P$. sidoides tem sido atribuída às propriedades antimicrobianas e imunomoduladoras que o extrato possui.

Matthys et al. (2003), realizaram um estudo randomizado, duplo-cego, placebocontrolado com 468 adultos com bronquite aguda, no qual, foram administrados EPs 7630 e a solução placebo 30 gotas três vezes ao dia durante um período de sete dias. A duração da doença foi significativamente menor para os pacientes tratados com EPs 7630 comparado com o placebo. Nos primeiros quatro dias do início do tratamento o efeito foi reconhecido em 53,6\% dos pacientes que administraram EPs 7630 comparado com 36,2\% dos pacientes tratados com placebo. Os eventos adversos ocorreram em 36 dos 468 pacientes, entre os eventos adversos o EPs 7630 teve 20/233 e o placebo teve 16/235. Todos os eventos foram avaliados como não graves, sendo assim os autores concluiram que EPs 7630 foi mais eficaz em comparação com o placebo no tratamento de bronquite aguda, sugerindo que EPs 7630 reduziu claramente os sintomas e encurtou a incapacidade para o trabalho por quase dois dias.

Em um estudo multicêntrico envolvendo 259 crianças com bronquite aguda tratados com $P$. sidoides pelo período de 6 a 13 dias, demonstrou que $80 \%$ dos indivíduos obtiveram melhora nos sintomas individuais e o tratamento foi bem tolerado em 96,5\% dos casos (MIGOWISKI et al., 2006).

Blochin et al. (1999) compararam o tratamento com EPs 7630 e a terapia convencional com acetilcisteina em 60 crianças com idades entre 6 a 12 anos. As 

impressa) ISSN 2358-9426 (versão eletrônica)

crianças foram distribuídas aleatoriamente em dois grupos. Um grupo recebeu EPs 7630 em forma líquida, 20 gotas em cada hora até 12 vezes ao dia entre o primeiro e segundo dia de tratamento, e a partir do terceiro ao sétimo dia foram administrados 20 gotas três vezes ao dia. O segundo grupo recebeu Acetilcísteina em forma de grânulos e foi administrada $200 \mathrm{mg}$ por dia durante um período de sete dias. Os resultados foram medidos através da mudança típica dos sintomas de bronquite aguda. Os sintomas específicos de bronquite foram reduzidos de forma mais eficaz e rápida com EPs 7630 do que o tratamento convencional com acetilcísteina.

A eficácia e tolerabilidade do EPs 7630 foram investigadas em um estudo prospectivo, aberto, multicêntrico em 205 pacientes que sofrem de bronquite aguda ou exacerbação dos sintomas de bronquite crônica. Os pacientes estudados eram maiores de 18 anos e foram administradas 30 gotas da solução EPs 7630 (a solução continha 80g de EPs 7630 em $100 \mathrm{~mL}$ de solução) três vezes ao dia, durante um período de sete dias. O principal resultado analisado foi à mudança na pontuação total de cinco sintomas típicos de bronquite (tosse, expectoração, chiado, dor no peito durante a tosse e dispnéia) e o início da ação surgiu em dois dias em média. Os eventos adversos ocorreram em 16 pacientes e estes não foram considerados graves. Os resultados do estudo confirmaram a eficácia do tratamento com EPs 7630 no tratamento de bronquite aguda e diminuição dos sintomas em $78 \%$ dos pacientes que possuíam bronquite crônica. Os pacientes mostraram-se satisfeitos ou muito satisfeitos com os resultados do tratamento (MATTHYS; HEGER, 2007).

\section{Infecções do Trato respiratório (atividade antibacteriana e imunomoduladora)}

Bereznoy et al. (2003) em um estudo prospectivo, randomizado, duplo-cego e controlado por placebo, avaliou o uso de P. sidoides (EPs 7630) em 143 crianças com idade entre 6 a 10 anos com diagnóstico de faringoamigdalite aguda não-estreptocócica do grupo A. Os resultados indicaram que o extrato de $P$. sidoides resultou numa melhora evidente em quase $90 \%$ das crianças, enquanto que no grupo placebo apenas em 17\%. Em relação à segurança apenas $1,4 \%$ das crianças tratadas com EPs 7630 apresentaram eventos adversos, sendo que no grupo placebo 20\%. O EPs 7630 apresentou tolerabilidade boa ou muito boa em $97,3 \%$ dos casos. 
Revista Tecnologia e Ambiente, v. 21, 2014, Criciúma, Santa Catarina. ISSN 1413-8131 (versão impressa) ISSN 2358-9426 (versão eletrônica)

Em 2005, Heger publicou um estudo multicêntrico, randomizado, duplo cego e controlado por placebo em 93 pacientes com resfriado comum, com idade entre 18 a 55 anos. Os pacientes receberam 30 gotas de $P$. sidoides ou placebo, três vezes ao dia durante dez dias. Os resultados deste estudo evidenciaram melhora nos sintomas dos pacientes como, por exemplo, congestão nasal, espirros, dor de garganta, rouquidão, dor de cabeça, tosse, dores musculares entre outras. Logo, P. sidoides comparado com placebo demonstrou boa eficácia e segurança no tratamento de resfriados comuns.

Foi realizado um estudo de Vigilância Epidemiológica conduzidos por Kirk e Netto (2007), com 1667 pacientes com idade entre 01 e 65 anos com objetivo de comprovar a segurança e efetividade de $P$. sidoides (Umckan ${ }^{\circledR}$ ) no tratamento das tonsilofaringites agudas presumidamente virais. Este estudo foi observacional, prospectivo, aberto, em crianças, adolescentes e adultos atendidos em consultórios e ambulatórios por todo o Brasil. Segundo critérios de avaliação estabelecidos pelos pesquisadores e descritos no estudo a tolerância de $P$. sidoides foi classificada como boa/muito boa por $95,9 \%$ dos médicos que o prescreveram e por $93,8 \%$ dos pacientes. Apenas 22 pacientes $(1,4 \%)$ apresentaram eventos adversos, principalmente diarréia, epigastralgia e exantema.

Em estudo in vitro, foi investigado se EPs 7630 possuía ação antiadesão a um grupo de Streptococcus do grupo A. Segundo o autor EPs 7630 desenvolveu propriedades antibacterianas, pois o mesmo reduziu à aderência bacteriana as células epiteliais intactas, protegendo o organismo contra a colonização e infecção bacteriana. $\mathrm{O}$ autor ressalta que o extrato é uma ótima alternativa para tratar infecções das vias aéreas superiores e consequentemente evitar o uso de antibióticos desnecessários que podem se tornarem resistentes (CONRAD et al., 2007b).

Mativandlela et al. (2006), avaliaram a atividade antibacteriana, antifúngica e antitubercular in vitro do extrato etanólico das raízes de $P$. sidoides. O extrato inibiu o crescimento de Haemophilus influenzae, Moraxella catarrhalis, Mycobacterium tuberculosis, Aspergillus niger, Fusarium oxysporum e Streptococcus pneumoniae em uma concentração de $5 \times 10^{-3} \mathrm{mg} / \mathrm{L}$.

A eficácia do extrato de P. sidoides contra o Herpesvirus (HSV-1 e HSV-2) foi estudado in vitro por Schnitzler et al. (2008). O estudo demonstrou uma inibição de 99,9\% tanto em HSV-1 quanto HSV-2, sugerindo que o extrato de P. sidoides poderia 

impressa) ISSN 2358-9426 (versão eletrônica)

ser um tratamento antiviral promissor para infecção por herpes simples e por herpes genital.

Já Koldziej e Kiderlen (2007), em estudo in vitro confirmaram que o EPs 7630 possui uma fraca atividade antibacteriana sobre diferentes bactérias Gram positivas e Gram negativas. Os efeitos sobre a resposta imune não específica também tem sido demonstrados, indicando que $P$. sidoides tem propriedades imunomoduladora e imunorestauradora. Dentre os mecanismos de estímulo da resposta imune inespecífica destacam-se o estímulo da atividade de células natural killers, modulação da síntese de interferons e citocinas, estímulo da frequência dos batimentos ciliares das células epiteliais e estímulo da atividade fagocitária.

Neugebauer et al. (2005), avaliaram se EPs 7630 possuía a capacidade de aumentar a frequência dos batimentos ciliares em culturas de células nasais in vitro. A frequência dos batimentos ciliares aumentaram na concentração de 30 (123\%) e 100 (133\%) $\mathrm{mg} / \mathrm{mL}$ sugerindo que o extrato de $P$. sidoides é uma ótima alternativa para tratar doenças das vias respiratórias superiores. Embora as substâncias ativas responsáveis pelo aumento da frequência dos batimentos ciliares ainda são desconhecidas, novos estudos serão necessários para fornecer dados sobre os componentes ativos de EPs 7630.

Vários estudos in vitro relacionados com a atividade antibacteriana e imunomoduladora confirmam que o extrato de $P$. sidoides tem excelente perfil farmacológico para tratar infecções do trato respiratório (CONRAD et al., 2007a; SCHÖTZ; NÖLDNER, 2007; TRUN et al., 2006).

\section{Doenças gástricas}

A raiz de $P$. sidoides vem sendo utilizada há muitos anos na medicina tradicional em zonas da África do Sul para tratamento de distúrbios gastrointestinais (TRUN et al., 2006).

Em um estudo in vitro realizado por Beil e Kilian (2007), foi avaliada a atividade antibacteriana do EPs 7630 contra a Helicobacter pylori em células epiteliais gástricas. Os resultados demonstraram que EPs 7630 inibiu o crescimento e aderência bacteriana da Helicobacter pylori nas células epiteliais gástricas. Outro estudo 

impressa) ISSN 2358-9426 (versão eletrônica)

evidenciou que o extrato da raiz de $P$. sidoides é um potente agente antiadesão e pode ser uma opção útil para prevenir a ação dessa bactéria (WITTSCHIER et al., 2007).

Vários compostos estão sendo avaliados para determinar quais são os constituintes químicos responsáveis pela ação antiadesão de $P$. sidoides contra a $H$. pylori. Uma possibilidade é que as proantocianidinas sejam responsáveis pela ação, entretanto se faz necessário aprofundar os estudos (WITTSCHIER et al., 2007).

\section{Posologia do extrato de Pelargonium sidoides (EPs 7630)}

Segundo informações disponíveis na bula do medicamento fitoterápico preparado com raízes de $P$. sidoides $\left(\right.$ Kaloba $\left.^{\circledR}\right)$, para tratar infecções do trato respiratório, este fitomedicamento deve ser administrado por via oral, com pequena quantidade de líquido, meia hora antes das refeições. Para infecções agudas, adultos e crianças maiores de 12 anos devem ingerir 30 gotas, três vezes ao dia, já crianças com idade entre 6 e 12 anos, se administra 20 gotas, três vezes ao dia e crianças menores de 6 anos 10 gotas, três vezes ao dia.

Em caso de evolução crônica da doença ou recorrência frequente, a dose a ser administrada em adultos e crianças maiores de 12 anos é de 20 gotas três vezes ao dia. A duração média de tratamento é de 5 a 7 dias e não deve exceder três semanas, sendo a concentração do medicamento/mL de $825 \mathrm{mg}$ de extrato etanólico das raízes de $P$. sidoides $/ \mathrm{mL}$.

\section{Efeitos adversos}

Agbabiaka et al. (2008) conduziram um estudo de revisão e metanálise com $P$. sidoides para tratamento de bronquite aguda onde foram avaliados cinco ensaios clínicos randomizados e não foram relatados eventos adversos graves. No entanto, alguns dos efeitos adversos relatados incluem perturbações gastrointestinais, tais como diarreia e epigastralgia devido o extrato de $P$. sidoides possuir alto teor de taninos.

Uma notificação de obstipação e oligúria foi relatado a um Centro de Farmacovilância, porém o paciente usava concomitantemente um xarope de Hedera helix (BALBINO; DIAS, 2010). Também há relatos de reações alérgicas, sangramento 
Revista Tecnologia e Ambiente, v. 21, 2014, Criciúma, Santa Catarina. ISSN 1413-8131 (versão impressa) ISSN 2358-9426 (versão eletrônica)

discreto do nariz $(0,01$ a $0,1 \%$ dos pacientes) e em menos de $0,01 \%$ dos pacientes que utilizaram $P$. sidoides ocorreu elevação das enzimas hepáticas, mas a relação causal entre essa reação ao uso não foi demonstrada (ADIS INTERNATIONAL LTDA, 2006).

\section{Interações medicamentosas}

Devido ao teor de cumarinas, presentes no extrato de $P$. sidoides, há discussões sobre risco de hemorragia se usado com anticoagulantes derivados da cumarina como, por exemplo, o fármaco Varfarina (KOCH; BIBER, 2007; DE BOER et al., 2007).

No entanto, Koch e Biber (2007), em estudo in vivo com ratos, avaliaram a farmacocinética do uso concomitante de EPs 7630 com a Varfarina. O estudo indicou que não houve influência significativa sobre parâmetros da coagulação com a administração concomitante da Varfarina com EPs 7630, quando comparado com os animais tratados somente com a Varfarina. Entretanto, não há estudos em humanos que comprovem os mesmos resultados.

\section{Contraindicações}

$P$. sidoides não deve ser usado durante a gravidez e lactação e em pacientes com maior tendência à hemorragia ou doenças hepáticas e renais graves, por não haver experiência clínica nestes casos e em casos de hipersensibilidade aos componentes da fórmula. A administração concomitante de derivados cumarínicos pode, teoricamente, aumentar a ação anticoagulante (KIRK; NETTO, 2007).

\section{Toxicidade}

Quanto à segurança, $P$. sidoides tem-se mostrado bastante seguro, sendo que sua DL50 foi estimada em 48,5 mL/kg de peso corporal, aproximadamente 1000 vezes a dose terapêutica diária recomendada. Além disso, a solução de P. sidoides EPs 7630 vem sendo comercializada na Alemanha há mais de 50 anos. Entre 1994 e 2000, foram vendidas 63,5 milhões de doses diárias e um número significativo de pacientes tratados 

impressa) ISSN 2358-9426 (versão eletrônica)

foram crianças. Nenhum evento adverso grave foi notificado, o que representa uma experiência de segurança favorável a este produto (MIGOWISKI et al., 2006).

Estudo de vigilância pós-comercialização, realizado em 2007 em território nacional brasileiro, também confirmou excelente perfil de segurança com uma taxa de eventos adversos de 1,4\% (KIRK; NETO, 2007).

\section{CONSIDERAÇÕES FINAIS}

As plantas medicinais vêm sendo utilizadas na terapêutica há milhares de anos e o seu uso popular foi propagado de geração em geração e descrito em diversas farmacopéias e literaturas oficiais. Atualmente, o interesse pelas terapias naturais ou complementares, dentre elas a fitoterapia, está associado ao fato de que a população busca melhor qualidade de vida, questionando quanto aos perigos do uso abusivo e irracional dos medicamentos sintéticos procurando substituí-los por fitoterápicos e plantas medicinais.

Neste contexto, destaca-se Pelargonium sidoides DC., planta medicinal nativa do sul da África, popularmente conhecida na região como Umckaloabo e com amplas indicações populares. Dentre elas, destaca-se o uso para infecções do trato respiratório, principalmente para o tratamento de bronquite aguda, sendo estas indicações comprovadas e avaliadas quanto sua eficácia e segurança para o uso em seres humanos como medicamento fitoterápico.

Sua validação contribui positivamente para as indicações do uso de $P$. sidoides na prática médica. No mercado farmacêutico brasileiro os fitoterápicos registrados na ANVISA com esta planta, ambos produzidos com o extrato padronizado "EPs 7630", é composto por vários constituintes químicos, dentre eles as cumarinas e os polifenóis (taninos), que segundo os estudos são os principais componentes responsáveis pelos efeitos terapêutico do extrato.

$P$. sidoides representa uma alternativa valiosa devido ao seu efeito antibacteriano e por reforçar a imunidade não específica, consequentemente pode contribuir positivamente para a redução das prescrições de antibióticos desnecessários nas infecções virais das vias aéreas superiores e diminuir os riscos de resistência bacteriana na prática clínica. Além desses efeitos, P. sidoides demonstrou in vitro aumento dos 
Revista Tecnologia e Ambiente, v. 21, 2014, Criciúma, Santa Catarina. ISSN 1413-8131 (versão impressa) ISSN 2358-9426 (versão eletrônica)

batimentos ciliares nas mucosas nasais, sugerindo que também possa aumentar a função protetora contra infecções bacterianas. Destaca-se ainda a ausência de toxicidade e baixo potencial de efeitos adversos, tanto para adultos como para crianças.

Embora com todas as vantagens descritas acima, seu uso como medicamento é recente no mercado nacional e muitos profissionais desconhecem seus benefícios terapêuticos. Espera-se que este trabalho contribua para a divulgação e conhecimento desta alternativa terapêutica, para o tratamento das infecções do trato respiratório, entre os profissionais da área da saúde e favoreça sua utilização racional, além de instigar futuras pesquisas com esta planta.

\section{REFERÊNCIAS BIBLIOGRÁFICAS}

ADIS INTERNATIONAL LTDA. Monografia Kaloba, Pelargonium sidoides Extrato EPs 7630. São Paulo: Altana Pharma Ltda, 2006. 44 p. Disponível em: 〈http://www.nycomedpharma.com.br/nycomed_brasil/Kaloba_monografia.pdf $>$.

Acesso em: 10 ago. 2008.

AGBABIAKA, T.B.; GUO, R.; ERNST, E. Pelargonium sidoides for acute bronchitis: a systematic review and meta-analysis. Phytomedicine, Vellberg, v. 15, n. 5, p. 378385, 2008.APG III. The Angiosperm Phylogeny Group. An update of the Angiosperm Phylogeny Group classification for the ordens and families of flowering plants: APG III. Botanical Journal of the Linnean Society, London, v. 161, p. 105-121, 2009.

ASCHWANDEN, C. Herbs for health, but how safe are they? Bull. W. H. O., Genebra, v. 79, n. 7, p. 691-692, 2001.

BALBINO, E. E.; DIAS, M. F. Farmacovigilância: um passo em direção ao uso racional de plantas medicinais e fitoterápicos. Rev. Bras. Farmacogn., Curitiba, v.20, n.6, p. 992-1000, 2010.

BEIL,W.; KILIAN,P. EPs 7630, an extract from Pelargonium sidoides roots inhibits adherence of Helicobacter pylori to gastric epithelial cells. Phytomedicine, Velberg, v. 14, n. 6, p. 5-8, 2007.

BEREZNOY, V.V.; RILEY, D.S.; WASSMER, G.; HEGER, M. Efficacy of extract of Pelargonium sidoides in children with acute non-group A beta-hemolytic streptococcus tonsillopharyngitis: a randomized, double-blind, placebo-controlled trial. Altern Ther Health Med, Aliso Viejo, v. 9, n.5, p. 68-79, 2003.

BLADT, S.; WAGNER, H. From the Zulu medicine to the European phytomedicine Umckaloabo. Phytomedicine, Velberg, v.14, n.1, p. 2-4, 2007.

BLOCHIN, B, HAIDVOGL, M, HEGER, M. Umckaloabo im Vergleich zu Acetylcystein bei Kindern mit acuter Bronchitis: prospektive, randomisierte, kontrollierte, offene Studie zur Wirksamkeit und Vertra glichkeit. Der Kassenarzt, Karlsrhe, v. 49, p. 46-50, 1999. 
Revista Tecnologia e Ambiente, v. 21, 2014, Criciúma, Santa Catarina. ISSN 1413-8131 (versão impressa) ISSN 2358-9426 (versão eletrônica)

BRASIL. Agência Nacional de Vigilância Sanitária. (ANVISA). Registro do medicamento Kaloba. Brasília (D.F). 2004. Disponível em: <http://www7.anvisa.gov.br/datavisa/Consulta_Produto/rconsulta_produto_internet.as $>$. Acesso em: 15 set. 2008.

BRASIL. Agência Nacional de Vigilância Sanitária. (ANVISA). Registro do medicamento Umckan. Brasília (D.F). 2007. Disponível em: <http://www7.anvisa.gov.br/datavisa/Consulta_Produto/rconsulta_produto_internet.as $>$. Acesso em: 15 set. 2008.

BRASIL. Agência Nacional de Vigilância Sanitária. (ANVISA). RDC n 26 de 13 maio de 2014. Dispõem sobre o Registro de Medicamentos Fitoterápicos e o Registro e a Notificação de Produtos Tradicionais Fitoterápicos. Brasília (D.F), 2014

CASTRO, A., VLOK, J.H., NEWTON, D., MOTJOTJI, L. \& RAIMONDO, D. Pelargonium sidoides DC. National Assessment: Red List of South African Plants. 2012. Disponível em: <http://redlist.sanbi.org/species.php?species=1976-307>.Acesso em: 20 out. 2014.

CONRAD, A.; HASMANN, C.; ENGELS, I.; DASCHNER, F.D.; FRANK, U.. Extract of Pelargonium sidoides (EPs 7630) improves phagocytosis, oxidative burst, and intracellular killing of human peripheral blood phagocytes in vitro. Phytomedicine, Velberg, v.14, n. 1, P. 46-51, 2007a.

CONRAD, A.; JUNG, I.; LALLEMAND, C.; CARRAPATOSO, F.; ENGELS, I.; DASCHNER, F.D.; FRANK, U. . Extract of Pelargonium sidoides (EPs 7630) inhibits the interactions of group A-streptococci and host epithelia in vitro. Phytomedicine, Velberg, v. 14, n. 6, p.52-59, 2007 b.

DE BOER, H.J.; HAGEMANN, U.; BATE, J.; MEYBOOM, R.H.. Allergic reactions to medicines derived from Pelargonium species. Drug Saf, Maryland, v. 30, n. 8, p. 677680, 2007.

GOLOVATIOUK, A.; CHUCHALIN, A.G. Efficacy of an extract from Pelargonium sidoides (EPs 7630) compared to placebo in patients with acute bronchitis. Phytopharmaka, Darmstadt, v. 7, p. 1-7, 2002.

HEGER, M. Efficacy and safety of an extract of Pelargonium sidoides (EPs) in the treatment of the common cold. A multi-center, double- blind, placebo-controlled trial. Presentacion de poster. In: THE FIRST INTERNACIONAL CONFERENCE ON NATURAL PRODUCTS AND MOLECULAR THERAPY, 2005. Cape Town, South Africa. Anais... New York: New York Academy of Sciences, 2005. p. 12-14.

KALOBA: solução oral- Fitoterápico. Responsável técnico Wagner Moi. JaguariúnaSP: Altana Pharma Ltda. Bula de remédio, 2004.

KIRK, K. M.; NETTO, P. G. G.. Vigilância pós-comercialização do Pelargonium sidoides no tratamento das tonsilofaringites comunitárias agudas de origem presumidamente viral. Rev Panam Infectol, São Paulo, v. 9, n. 1, p. 15-24, 2007.

KOCH, E.; BIBER, A. Treatment of rats with the Pelargonium sidoides extract EPs 7630 has no effect on blood coagulation parameters or on the pharmacokinetics of warfarin. Phytomedicine, Velberga, v. 14, n. 6, p. 40-45, 2007. 
KOLODZIEJ, H. Aqueous ethanolic extract of the roots of Pelargonium sidoides-new scientific evidence for an old anti-infective phytopharmaceutical. Planta.Med, Antwerp, v.74, n.6, p. 661-666, 2008.

KOLODZIEJ, H. ; KAYSER, O.; RADTKE, O. A.; KIDERLEN, A. F.; KOCH, E. Pharmacological profile of extracts of Pelargonium sidoides and their constituents. Phytomedicine, Velberga, v. 10, n. 4, p. 18-24, 2003.

KOLODZIEJ, H. Fascinating metabolic pools of Pelargonium sidoides and Pelargonium reniforme, traditional and phytomedicinal sources of the herbal medicine Umckaloabos. Phytomedicine, Velberg, v. 14, n. 6, p. 9-17, 2007.

KOLODZIEJ, H.; KIDERLEN, A. F. In vitro evaluation of antibacterial and immunomodulatory activities of Pelargonium reniforme, Pelargonium sidoides and the related herbal drug preparation EPs 7630. Phytomedicine, Velberg, v. 14, n.6, p. 18-26, 2007.

LEWU, F. B.; GRIERSON, D. S.; AFOLAYAN, A. J. Clonal propagation of Pelargonium sidoides: A threatened medicinal plant of South Africa. African Journal of Biotechnology, Nigéria, v. 5, n. 2, p. 123-125, 2006.

LORENZI, H.; MATOS, F. J. A. Plantas medicinais no Brasil: nativas e exóticas. São Paulo: Instituto Plantarum, 2002. 512 p.

MATIVANDLELA, S. P. N; LALL, N.; MEYER, J. J. M. Antibacterial, antifungal and antitubercular actvity of (the roots of) Pelargonium reniforme (CURT) and Pelargonium sidoides (DC) (Geraniaceae) root extracts. South African Journal of Botany, Pietermaritzburg, v. 72, n.2, p. 232-237, 2006.

MATTHYS, H.; EISEBITT, R.; SEITH, B.; HEGER, M. Efficacy and safety of an extract of Pelargonium sidoides (EPs 7630) in adults with acute bronchitis. A randomised, double-blind, placebo-controlled trial. Phytomedicine, Velberg, v. 10, n.4, p. 7-17, 2003.

MATTHYS, H.; KAMIN, W.; PUNK, P.; HEGER, M. Pelargonium sidoides preparation (EPs 7630) in the treatment of acute bronchitis in adults and children. Phytomedicine, Velberg, v. 14, n.1, p. 69-73, 2007.

MATTHYS, H; HEGER, M. EPs 7630-solution an effective therapeutic option in acute and exacerbating bronchitis. Phytomedicine, Velberg, v. 14, n.1, p. 65-68, 2007.

MIGOWISKI, E; ZILLI, R; REIS, B. Pelargonium sidoides: uma alternativa nas infecções do trato respiratório. Somerj sociedade brasileira do Rio de Janeiro, Botafogo, $\quad$ v. $4, \quad 2006.4$ Disponível <http://www.somerj.com.br/revista/200604/2006_04__artigocientifico.htm>. Acesso em: 05 maio. 2008.

NEUGEBAUER, P.; MICKENHAGEN, A.; SIEFER, O.; WALGER, M. A new approach to pharmacological effects on ciliary beat frequency in cell cultures exemplary measurements under Pelargonium sidoides extract (EPs 7630). Phytomedicine, Velberg, v. 12, 1-2, p. 46-51, 2005.

NÖLDNER, M.; SCHÖTZ, K. Inhibition of lipopolysaccharid-induced sickness behavior by a dry extract from the roots of Pelargonium sidoides (EPs 7630) in mice. Phytomedicine, Velberg, v. 14, n.1, p. 27-31, 2007. 
Revista Tecnologia e Ambiente, v. 21, 2014, Criciúma, Santa Catarina. ISSN 1413-8131 (versão impressa) ISSN 2358-9426 (versão eletrônica)

SCHNITZLER, P.; SCHNEIDER, S.; STINTZING, F. C.; CARLE, R.; REICHLING, J. Efficacy of an aqueous Pelargonium sidoides extract against herpesvirus. Phytomedicine, Velberg, v. n. 12, p. 1108-1116 2008.

SCHOETZ, K.; ERDELMEIER, C.; GERMER, S.; HAUER, H. A detailed view on the constituents of EPs 7630. Planta Med, New York, v.74, n.6, p.667-674, 2008.

SCHOTZ, K.; NOLDNER, M. Mass spectroscopic characterisation of oligomeric proanthocyanidins derived from an extract of Pelargonium sidoides roots (EPs 7630) and pharmacological screening in CNS models. Phytomedicine, Velberga,v. 14, n.1, p. 32-39, 2007.

SEIDEL, V.; TAYLOR, P. W. In vitro activity of extracts and constituents of Pelagonium against rapidly growing mycobacteria. Int J Antimicrob Agents, Birmingham, v. 23, n. 6, p. 613-619, 2004.

SIMÕES, C. M. O.; SCHENKEL, E. P.; GOSMANN, G.; MELLO J. C. P.; MENTZ, L. A.; PETROVICK, P. R. (Org.). Farmacognosia: da planta ao medicamento. 6. ed.rev. ampl. Porto Alegre, Florianópolis: Universidades UFRGS/ UFSC, 2010, 1102 p.

TOMAZZONI, M. I.; NEGRELLE, R. R. B.; CENTA, M. L. Fitoterapia popular: a busca instrumental enquanto prática terapêutica. Texto \& Contexto Enfermagem, Florianópolis, v. 15, n.1, p. 115-121, 2006.

TRUN, W.; KIDERLEN, A. F.; KOLODZIEJ, H. Nitric oxide synthase and cytokines gene expression analyses in Leishmania-infected RAW 264.7 cells treated with an extract of Pelargonium sidoides (EPs 7630). Phytomedicine, Velberg, v. 13, n.8, p. 570-575, 2006.

TUROLLA, M. S. R.; NASCIMENTO, E. S. Informações toxicológicas de alguns fitoterápicos utilizados no Brasil. São Paulo. Rev. Bras. Cienc. Farm, São Paulo, v. 42, n. 2, p. 289-306, 2006.

VAN DER WALT, J.J., VORSTER, P.J. AND WARD-HILHORST, E. Pelargoniums of Southern Africa. Kirstenbosch: National Botanic Gardens, 1988.

VAN WYK, B. E. A broad review of commercially important southern African medicinal plants. J Ethnopharmacol, Leiden, v. 119, n. 3, p. 1-14, 2008.

VLOK, J. Assessment of Veld Harvesting of Pelargonium sidoides and Pelargonium reniforme in the Eastern Cape - First survey report. Wellington: Parceval (Pcy) Ltd, 2003. P.27. Repot commissioned, South Africa.

WHITE, A.G. The effect of geography, cultivation and harvest technique on the umckalin concentration and growth of Pelargonium sidoides (Geraniaceae). 2007. 144 f. Tese. (Doutorado em Ciências em Botânica) - Rhodes University da África do Sul, África do Sul, 2007.

WITTSCHIER, N; FALLER, G.; HENSEL, A. An extract of Pelargonium sidoides (EPs 7630) inhibits in situ adhesion of Helicobacter pylori to human stomach. Phytomedicine, Velberg, v. 14, n.4, p. 285-288, 2007. 